\section{Cystine Requirements of Fleece Growth}

THE considerable sulphur content of clean wool, and its presence substantially in the form of a cystine nucleus, has led to much inquiry into the question of cystine being a limiting factor in wool growth. Various estimates of the cystine requirement for the fleece production have been made, on the assumption that this is given by the quantity of cystine recover. able in the wool.

Of the total cystine intake, a certain proportion is katabolised, and metabolised other than in follicle activity, but certain estimates have indicated that the total intake so nearly reaches the wool cystine yield (and in some estimates is actually exceeded by it), that Rimington and Bekker ${ }^{1}$ have suggested that the sheep is an exception to the generally accepted view that cystine must be preformed in the animal diet. This is rejected by Woodman and Evans ${ }^{2}$, who claim that their pasture analyses, quoted by Rimington and Bekker, provide, on their interpretation, an adequacy of cystine for the clean wool yield.

It seems clear that the present available methods for cystine estimation, as applied to foodstuffs, are too uncertain to permit of any definite conclusion on this fundamental point. Also it has apparently not been realised that it is fallacious to regard the cystine content of the wool as being identical with that utilised by the follicle population, so that it cannot therefore be regarded even as a minimal requirement.

The follicle mechanism is as much concerned in elaborating the inner root sheath as the fibre proper. In fact, on the cystine gradient postulated by us ${ }^{3}$, the inner root sheath would have priority in call upon the cystine supply. This inner root sheath is dissipated by fragmentation, and goes to join other products, for example, wool fat and suint, which form the non-wool fraction of the whole fleece, and is therefore excluded from the chemical analysis of the clean wool.

With certain observed cases of diminished fibre growth, accumulation of this inner root sheath material (no doubt contaminated with ordinary epiderrnal 'scurf') has occurred, which has provided sufficient material for qualitative tests. It gives, with Sullivan's reagent and the lead acetate test, strongly positive reactions for cystine. (Also the Pauly test reacts positively for tyrosine.)

It is hoped to determine with more precision the cystine content of this material ordinarily lost; meanwhile, however, it would not be unreasonable to assume that it is at least no less than that of the fibre proper.

In any event, the cystine absorbed in inner root sheath formation, and ultimately disappearing from the food cystine-wool cystine balance sheet, becomes significant in a degree depending upon the relative rates of production of inner root sheath and fibre. Approximate estimates of these relative proportions are being sought.

Textile Department,

A. T. KING.

The University,

Leeds.

J. E. NichoLs,

Wool Industries Research Association, Torridon, Leeds. Nov. 15.

1 Rimington and Bekker, Nature, 129, 687, May 7, 1932.

${ }^{2}$ Woodman and Evans, NATURE, 130, 1001, Dec. 31, 193

${ }^{3}$ King and Nichols, Trans. Faraday Soc., 29, 272 ; 1933.
Acceleration of Tissue Respiration by a Nitrophenol

WHILsT it has been known since 1885 that certain nitrophenols can cause a remarkable increase in the metabolism of the whole animal, the mechanism of this action is still little understood. Experiments with perfused limbs led Magne, Mayer and Plantefol $^{1}$ to the view that $1: 2: 4$-dinitrophenol ('thermol') causes a direct stimulation of cellular oxidations with increased carbohydrate consumption. Proof of this idea is now given by the experiments described below, in which it is shown that a nitrophenol can cause a large increase in the respiration of thin slices of surviving rat tissue when added in suitable concentration to the medium in which they are respiring

The measurements were made in the HaldaneBarcroft-Warburg apparatus, and respiratory quotients were determined in bicarbonate-containing salt solutions by the method of Dickens and Simer ${ }^{2}$. The nitrophenol used was 4:6-dinitro-o-cresol, this being pharmacologically the most active body of this type so far tested (Dodds and Pope ${ }^{3}$ ).

Dinitro-o-cresol increases the respiration of surviving kidney tissue. According to Barron ${ }^{4}$, only those tissues which have an aerobic glycolysis show an increased respiration in the presence of the reversible dyestuff methylene blue. Kidney has a very low aerobic glycolysis, and its respiration in glucose is slightly decreased by methylene blue. Yet dinitro-0-cresol in $10^{-5} \mathrm{~m}$. concentration will cause increases of respiration up to 80 per cent with kidney in lactate-containing phosphate media. The respiration is maintained at the increased value for at least one hour following addition of the nitrobody. Under these conditions, the $Q_{\mathrm{O}_{2}}$ may rise to so high as 60 , that is, to about double the normal value for rat retina in glucose. Respiratory quotient determinations show that the extra oxygen uptake caused by the presence of the nitro-body represents complete oxidation of foodstuff, as is shown by the following experiment with rat kidney slices in lactate-containing bicarbonate medium :

\begin{tabular}{|c|c|c|c|}
\hline & R.Q. & $\mathrm{QO}_{\mathrm{g}}$ & $Q_{\mathrm{CO}}^{\mathrm{O}_{2}}$ \\
\hline $\begin{array}{l}\text { Control } \\
\text { With } 10^{-5} \mathrm{M} \text {. }\end{array}$ & $0.78 ; 0.78$ (duplicates) & $-28.7 ;-29 \cdot 6$ & $-3 \cdot 4 ;-4 \cdot 6$ \\
\hline 0 -cresol & $0.87 ; 0.86$ (duplieates) & $-46 \cdot 0 ;-46 \cdot 7$ & -8.6 ; \\
\hline
\end{tabular}

Taking means of the duplicate determinations, the extra oxygen uptake due to the dinitro- 0 -cresol was $Q_{\mathrm{O}_{2}}=17 \cdot 2$; whilst the extra carbon dioxide production was $Q_{\mathrm{CO}_{2}}=17 \cdot 2$. Hence the extra oxygen burnt the metabolite at R.Q. unity. With rat brain cortex, which normally has $R . Q$. unity, the respiration was still maintained at a carbohydrate quotient when the oxygen uptake was increased about 40 per cent by dinitro-o-cresol. Respiration is also increased in pyruvate-containing media, being maintained at the theoretical $R . Q$. for pyruvate oxidation $(1 \cdot 2)$.

The respiration of kidney in the presence of glucose is increased in bicarbonate but not in phosphate-containing media. Whilst work is being undertaken to elucidate this surprising difference, it should be mentioned that Friedheim ${ }^{5}$ found with pyocyanine quite the reverse effect. With this catalyst the respiration of kidney was increased in phosphate but not in bicarbonate Ringer, but the magnitude of the increase is not stated.

$10^{-5} \mathrm{~m}$. appears to be roughly the optimal concentration for dinitro-o-cresol. With $10^{-4} \mathrm{~m}$. and higher concentrations the respiration is inhibited. 